\title{
Diversity of endogenous avian leukosis virus subgroup $E$ (ALVE) insertions in indigenous chickens
}

\author{
Andrew S. Mason ${ }^{1,2^{*}}$ (D), Katarzyna Miedzinska², Adebabay Kebede ${ }^{3,4}$, Oladeji Bamidele ${ }^{5}$, Ahmed S. Al-Jumaili, ${ }^{6,7}$
} Tadelle Dessie ${ }^{3}$, Olivier Hanotte ${ }^{3,6,7}$ and Jacqueline Smith²

\begin{abstract}
Background: Avian leukosis virus subgroup E (ALVE) insertions are endogenous retroviruses (ERV) that are restricted to the domestic chicken and its wild progenitor. In commercial chickens, ALVE are known to have a detrimental effect on productivity and provide a source for recombination with exogenous retroviruses. The wider diversity of ALVE in non-commercial chickens and the role of these elements in ERV-derived immunity (EDI) are yet to be investigated.

Results: In total, 974 different ALVE were identified from 407 chickens sampled from village populations in Ethiopia, Iraq, and Nigeria, using the recently developed obsERVer bioinformatics identification pipeline. Eighty-eight percent of all identified ALVE were novel, bringing the known number of ALVE integrations to more than 1300 across all analysed chickens. ALVE content was highly lineage-specific and populations generally exhibited a large diversity of ALVE at low frequencies, which is typical for ERV involved in EDI. A significantly larger number of ALVE was found within or near coding regions than expected by chance, although a relative depletion of ALVE was observed within coding regions, which likely reflects selection against deleterious integrations. These effects were less pronounced than in previous analyses of chickens from commercial lines.
\end{abstract}

Conclusions: Identification of more than 850 novel ALVE has trebled the known diversity of these retroviral elements. This work provides the basis for future studies to fully quantify the role of ALVE in immunity against exogenous ALV, and development of programmes to improve the productivity and welfare of chickens in developing economies.

\section{Background}

Retroviruses exhibit persistent yet highly changeable stress on their vertebrate hosts. Insertional mutagenesis can elicit a wide range of phenotypic effects and the rapidly evolving retroviral genome presents a constant immune challenge [1-3]. Furthermore, if a retrovirus integrates within the genome of the germline, these "endogenous" retroviruses (ERV) are inherited vertically, and can continue to affect the host organism over large evolutionary timescales. Thus, ERV provide a genomic

\footnotetext{
*Correspondence: andrew.mason@york.ac.uk

1 The University of York, York YO10 5DD, UK

Full list of author information is available at the end of the article
}

record of ancestral retroviral infections, and may elicit novel physiological stress by continuing to retrotranspose, produce retroviral proteins, and recombine, both across the genome and with exogenous retroviruses (Fig. 1) [3-9]. However, the effects of ERV are diverse, with some conferring resistance to new exogenous retroviral infections by three main strategies: receptor interference; inhibition of the retroviral lifecycle (uncoating, reassembly and nuclear localisation); and marking of retroviral RNA for degradation through formation of double stranded RNA [10-15]. Combined, these processes induce varying extents of ERV-derived immunity (EDI) in the host organism. EDI has been observed across vertebrates but elicits a largely transient response 


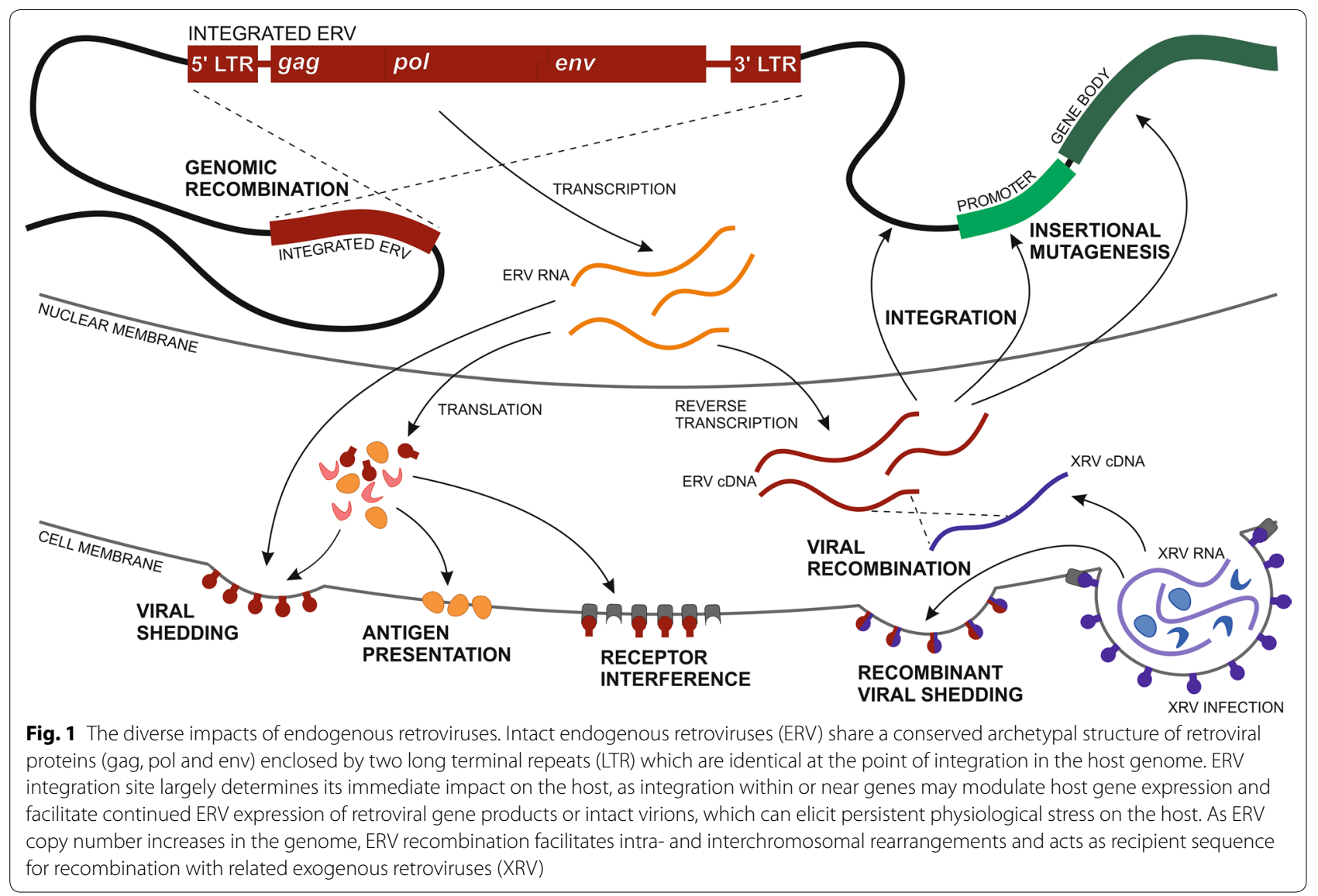

over evolutionary timescales, as ERV are retained while they confer a selective advantage and are then strongly selected against when that advantage is lost [2, 15-17].

In chickens (Gallus gallus), where ERV represent about $3 \%$ of the genome $[18,19]$, the only retrovirus with recurrent exogenous and endogenous activity is the avian leukosis virus (ALV) [20, 21]. ALV can infect all galliform birds, however subgroup E (ALVE) integrations are found only in the domestic chicken and its wild progenitor, the red junglefowl (RJF) [22]. ALVE have long been known to facilitate EDI [23, 24], but they have been primarily studied in commercial layer lines, where any selective benefit is masked by their typically negative association with productivity traits, and the absence of ALV infection in commercial stock [25-28].

A set of recent studies $[29,30]$ has begun to scratch the surface of true ALVE diversity within chickens, but primarily in commercial lines. A much broader characterisation of ALVE in non-commercial chickens is required to quantify the extent to which ALVE derive immunity to exogenous ALV. Furthermore, characterising the abundance of ALVE with known negative effects on productivity, or identifying novel ALVE that elicit positive effects on productivity or environmental adaptation, may lead to improvement of chicken meat and egg production in non-commercial settings. In this study, ALVE were identified in the genomes of 407 village chickens from Ethiopia, Iraq, and Nigeria to characterise ALVE diversity more comprehensively, and to assess the likely evolutionary and immunological significance of ALVE in a noncommercial setting.

\section{Methods}

\section{Animals and sequencing data}

Whole-genome (re)sequencing (WGS) data were analysed from 407 chickens (see Additional file 1) as part of the Centre for Tropical Livestock Genetics and Health (CTLGH) Poultry Genetics programme (http://www. ctlgh.org). Chickens were sourced from Ethiopia ( $\mathrm{n}=260$ from 25 populations), Iraq ( $\mathrm{n}=27$ from 3 populations) and Nigeria ( $\mathrm{n}=120$ from 14 populations). The sampled regions and numbers of sequenced individuals are summarised in Additional file 2: Table S1. Geographical data (altitude, vegetation cover, soil type) were available for each sampled region and phenotypic (weight, age, sex, relatedness, feather colour) and epidemiological (previous illnesses and treatment) data were recorded for individual chickens but were incomplete across all 
populations in each country, particularly in Nigeria and Iraq. All sequencing reads (Illumina $150 \mathrm{bp}$ paired-end) were quality-checked and trimmed where necessary [31-33].

\section{ALVE identification}

ALVE integrations were identified in the WGS data using the bioinformatics pipeline obsERVer, which has been used to identify ALVE in a wide range of chicken datasets [30]. Briefly, obsERVer maps WGS reads to an "ALVE pseudochromosome" that consists of 11 publicly available GenBank ALV sequences [30], extracts mapped reads and their read mates, and aligns these to the Gallus_gallus-5.0 chicken reference genome (Galgal5; GenBank: GCF_000002315.4), removing reads that map to assembled alpharetroviral integrations. A mapping quality greater than 20 was required for the pseudochromosome and reference genome alignments, and reads with secondary alignments within Galgal 5 were removed after filtering assembled alpharetroviral integrations. Putative ALVE integrations were annotated by known ALVE sites and manually validated after inspection using the Integrative Genomics Viewer (IGV) v2.4.3 software [34].

\section{Validation of identified ALVE integrations}

Previous validation of obsERVer-detected sites by PCRbased assays [30] showed high sensitivity with a false detection rate (FDR) of $0 \%$. However, given the diversity of the chicken populations in this study, and the high proportion of novel and lineage-specific ALVE, we performed additional validation. Twenty putative ALVE integration sites were selected at random from all novel ALVE integrations detected in this study to act as the validation set. For each of the 20 ALVE in the validation set, six sequenced individuals were chosen to represent the bioinformatically-predicted homozygous wildtype, homozygous integration, and heterozygous integration genotypes, where possible. Some individuals were used to validate multiple integrations (see Additional file 2: Table S2). Specific PCR assays were developed for each integration site using Primer3 v4.1.0 [35] (see Additional file 2: Table S3). PCR reactions were conducted using the Roche FastStart ${ }^{\mathrm{TM}}$ Taq DNA polymerase kit (Roche 04738357001) in $10 \mu \mathrm{l}$ reaction volumes with equal concentrations of primers. PCR began with an activation step at $95^{\circ} \mathrm{C}$ for $4 \mathrm{~min}$, followed by 35 cycles of $30 \mathrm{~s}$ denaturing at $95{ }^{\circ} \mathrm{C}, 30 \mathrm{~s}$ annealing at $60{ }^{\circ} \mathrm{C}$, and $45 \mathrm{~s}$ elongation at $72{ }^{\circ} \mathrm{C}$, with a final extension step at $72{ }^{\circ} \mathrm{C}$ for $7 \mathrm{~min}$. PCR products were detected on the Agilent 4200 TapeStation System using High Sensitivity D1000 ScreenTape (Agilent 5067-5584), following the manufacturer's instructions.

\section{ALVE distribution analysis}

All bioinformatically identified ALVE were combined to identify patterns in their genomic distribution. A dataset of an equal number of randomly generated insertions across Galgal 5 was used to identify any skews and biases in distribution, with the simulation repeated one million times. This simulated dataset was compared with the observed GC distribution for the target site duplication and windows of $100 \mathrm{bp}, 1 \mathrm{~kb}, 10 \mathrm{~kb}$, and $100 \mathrm{~kb}$ centred on the integration, and the distribution of ALVE relative to coding regions (Ensembl v87). Significant deviations across observed and simulated distributions were assessed with the two-sample Kolmogorov-Smirnov test, and between individual groups using a binomial test. Pearson correlations were derived between the ALVE distribution and $\log _{10}$ transformed values for assembled chromosome length, gene density, and chromosomelevel recombination rate (converted to Galgal5 from [36]). Significant deviation from the simulated data was assessed using the Fisher $z$-transformation.

\section{Direct ALVE genotyping and clustering}

Reads from each of the 407 datasets were mapped to Galgal5 using the BWA-mem v0.7.10 software [37], and the alignment maps were used to genotype each ALVE insertion. All identified ALVE were used for genotyping and all genotyping results correlated exactly with sites identified by obsERVer for each bird. A binary presence/ absence matrix for each ALVE within each individual was generated using 0 for the homozygous wild type and 1 for individuals that were homozygous at the ALVE insertion. This high dimension data was visualised using both $\mathrm{t}$-distributed stochastic neighbour embedding (t-SNE) [38] and hierarchical clustering with Jaccard distances, excluding the ALVE that were found in one individual only. Genotypes were correlated with available geographic, phenotypic and epidemiological data for each bird.

\section{Results and discussion \\ Distribution of ALVE across populations}

ALVE were detected from the WGS data of 407 individual chickens that were sampled from village populations in Ethiopia, Iraq, and Nigeria (see Additional file 2: Table S1). In total, 974 different ALVE were identified, with 6053 occurrences and an average of 14.9 ALVE per chicken. The number of ALVE per chicken was highly variable, ranging from six (comparable to levels in commercial brown egg layers [30]) to a maximum of 33. All populations across the three sampled countries exhibited a similar level of diversity. We identified 857 novel ALVE (88.0\%), which brings the known diversity of ALVE to 
over 1300 different integration sites [29, 30]. PCR assays were developed for 20 randomly selected novel ALVE integration sites (see Additional file 2: Table S2) to assess the obsERVer FDR, which was previously shown to be $0 \%$ in a commercial chicken dataset [30]. All selected integration sites were successfully validated by PCR (see Additional file 3: Figure S1), which confirmed an obsERVer FDR of $0 \%$ and that obsERVer is highly specific for the detection of ERV from WGS data.

Many of the previous ALVE detected in commercial chickens were also found in these indigenous chicken populations [29, 30,39]. However, it is unclear whether these represent the natural origins of these ALVE, or result from later introduction of Western commercial breeds. Among the identified ALVE, the commercially relevant ALVE21 was the most common. ALVE21 is a replication competent provirus that is associated with the sex-linked slow-feathering $K$ locus [40-42], and was present in $75 \%$ of all individuals and in all but one of the analysed populations (Dara Kumato, Ethiopia). ALVE1, ALVE3, ALVE15, ALVEB5 and ALVE-TYR were commonly found in all regions, as were ALVE_ros003, 010, 011, 159 and 276, which were previously identified in commercial layers and broilers, and a range of sites that were previously identified in two Ethiopian populations [30].

In total, 393 ALVE (40.3\%) were identified only in one individual and, within each population, 40 to $80 \%$ of the sites were detected in one bird only. This high diversity of low-frequency ALVE is typical of ERV-derived immunity (EDI), for which ERV are transiently beneficial to the host, since they provide resistance to new retroviral infections by receptor interference [15-17]. This has long been observed with the envelope protein of ALVE [23, $24,43,44]$, and with beta- and gammaretroviral ERV in mammalian species [10-12].

We found no ALVE that were fixed within a population, with the typical maximum ALVE population frequency ranging from 0.45 to 0.60 and a typical average frequency of 0.10 across all ALVE in a population. It is, however, possible that ALVE21 was fixed in seven of the analysed populations (see Additional file 1), in spite of the predominance of heterozygotes, caused by its presence in only one segment of the $K$ locus tandem repeat [30, 40]. Some of the homozygous ALVE21 genotypes may result from a reversion event at the $K$ locus [45], as was recently observed in commercial White Plymouth Rock layers [30, 46].

No significant associations with phenotypic or epidemiological data were identified for any ALVE or group of ALVE, although the metadata was incomplete. However, ALVE genotypes were sufficient to reconstitute the geographical distribution of the sampled chickens at the

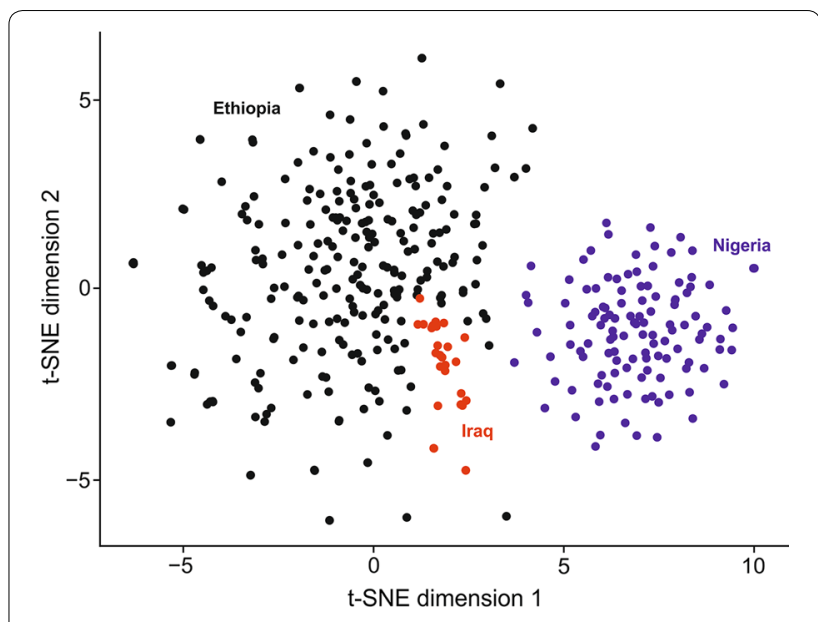

Fig. 2 t-SNE visualisation of the ALVE-resolved population structure of the sampled chicken populations. Dimension reduction was performed on a binary matrix of ALVE shared between at least two individuals $(n=581)$. Samples from each country are coloured black for Ethiopia, red for Iraq and blue for Nigeria. t-SNE was derived using sci-kit learn with Python 3.7 with a learning rate of 15 , perplexity of 65 , and a maximum of 10,000 iterations to ensure stability

national level (Fig. 2). The Iraqi samples were closely associated with those from the edge of the Ethiopian cluster, but the Nigerian populations were completely distinct, likely reflecting the relative geographical positions of the three countries. However, in most cases, we were not able to unambiguously resolve the population or regional level within each country based on ALVE genotypes alone (see Additional file 4: Figure S2). The relatively poor intra-national and predictable international resolution likely reflects the prevalence of trade within, rather than between, countries. It is possible that the resolution provided by ALVE genotypes is not sufficient to distinguish between closely related populations within a country, but that resolution could be improved by the incorporation of genetic variants that exist in larger numbers, such as single nucleotide variants (SNVs).

\section{Distribution of ALVE across the genome}

Integration of exogenous ALV occurs preferentially in open chromatin, particularly near protein-coding genes [47-49]. Although ALVE may exhibit the same biological preference, selection acts to remove deleterious endogenous elements over time. Accordingly, a recent analysis of ALVE in a dataset dominated by commercial chickens showed a significant depletion of ALVE within coding regions $(26.7 \%$ compared to $51.8 \%$ of modelled random integrations) but an eightfold enrichment of integrations within $10 \mathrm{~kb}$ of a protein-coding gene (32.9\% compared to $4.1 \%$ of modelled random integrations) [30]. Here, we observed a similar, but less extreme pattern of ALVE 


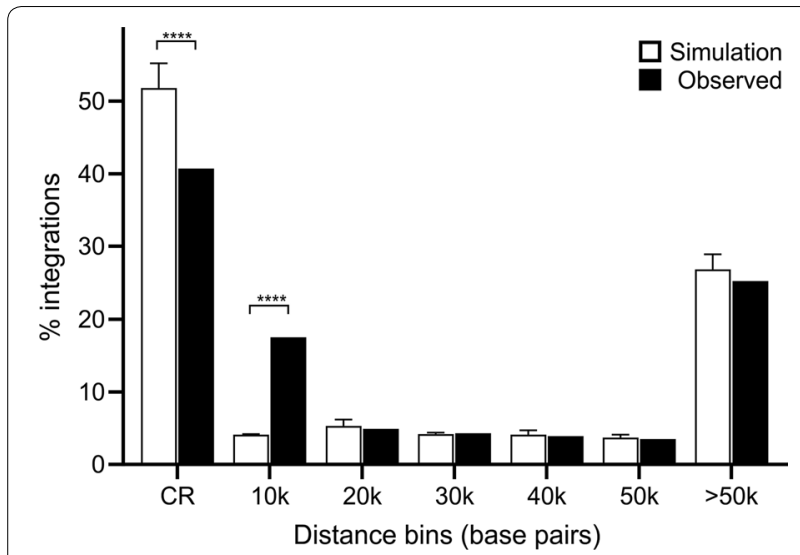

Fig. 3 ALVE distribution relative to coding features and randomly simulated integrations. Observed values represent all ALVE identified in this study $(n=974)$. Simulated values show the mean and standard deviation of one million randomly simulated redistributions of 974 integrations across the Galgal5 assembly. There was a significant depletion $\left(P=1.74 \times 10^{-14}\right)$ of integrations within coding regions (CR) and significant enrichment $\left(P=7.16 \times 10^{-19}\right)$ of integrations within $10 \mathrm{~kb}$ of CR. All other distance bins had non-significant differences. Specific values are reported in Additional file 2: Table S4

distribution (Fig. 3 and Additional file 2 Table S4), with 40.7\% of ALVE located within coding regions (depletion; $\mathrm{P}=1.74 \times 10^{-14}$ ) and $17.5 \%$ within $10 \mathrm{~kb}$ of protein-coding genes (enrichment; $\mathrm{P}=7.16 \times 10^{-19}$ ). These results likely reflect the much less intense selection of these village chickens compared to commercial chickens. Even with the apparent selection against integrations in coding regions, overall these data still indicate a significant enrichment of ALVE within or near protein coding genes $(P=0.03)$. This enrichment is also evidenced by the significantly elevated GC content of the ALVE target site duplications $\left(\mathrm{KS}=0.38 ; \mathrm{P}=7.22 \times 10^{-50}\right.$ ), although this effect was not observed for any other window size that was used for GC content calculation. Taken together, our results indicate that the distribution of ALVE is certainly not random. Given the structure of the chicken genome, ALVE density was highly correlated with chromosome length $\left(r=0.72 ; \mathrm{P}=3.03 \times 10^{-5}\right)$, but significantly less than expected with random integration $(r=0.97$; $\left.\mathrm{z}=26.56 ; \mathrm{P}=2.51 \times 10^{-154}\right)$. ALVE density had weaker, negative correlations with recombination rate and gene density. However, the variance in both these measures is largely explained by chromosome length $\left(\mathrm{r}^{2}=0.86\right.$ and $\mathrm{r}^{2}=0.83$, respectively).

\section{Integrations of ALVE within exons}

Only six of the 396 ALVE (1.5\%) found within coding regions were located in exons, which is significantly less than the $4.9 \%$ expected under random integration $\left(\mathrm{P}=6.36 \times 10^{-4}\right.$; see Additional file 2: Table S5). Two of these (ALVE_ros845 and ALVE_ros1003) were found in exon 4 of the pannexin1 (PANX1) gene, a gap junction family member that is expressed throughout the central nervous system. Both of these ALVE were identified in chickens from the Ethiopian Dibate region, and it is likely that the two sites have a shared history: they appear to be only 7 bp apart, but ALVE_ros 845 is associated with a genomic deletion that is likely to have a greater impact on PANX1 function. Two of the other exonic integrations were identified only in single individuals: ALVE_ros529 in the second exon of the cyclin dependent kinase 15 (CDK15) gene, which is known to regulate anti-apoptosis [50], and ALVE_ros586 in exon 4 of the IQ and ubiquitin-like domain-containing protein (IQUB) gene, which is involved in the regulation of cilia and the hedgehog signalling pathway [51]. Interestingly, both ALVE_ros569 and ALVE_ros638 were identified in individuals from different regions, with the former found only in one individual from Nigeria and one from Ethiopia. ALVE_ros569 is in exon 2 of the threonine synthase-like 2 (THNSL2) gene and may influence the ability of the bird to elicit an appropriate inflammatory response [52, 53], which is particularly relevant during persistent, ALVE-induced viremia. ALVE_ros638 may also influence response to viral load and regulation of anti-apoptosis due to its integration in exon 8 of the multidrug resistance-associated protein 6 (ABCC6, encoding the MRP6 protein) gene, however the distinct roles of MRP6 and a closely related truncated duplicate (URG7) are yet to be fully resolved [54].

It is also possible that integration of ALVE in these particular genes reflect a degree of selection (particularly with some sites in distant populations), as each affected gene is part of a large network with multiple redundancies. It would be of great interest to study the specific effects of exonic ALVE insertions, and to identify whether such integrations are tolerated by the host or actively selected against.

\section{Conclusions}

This study is the first step towards characterization of the diversity of ALVE that are present in non-commercial chickens. We identified 857 novel ALVE from a survey of more than 400 indigenous chickens from Ethiopia, Iraq, and Nigeria and observed a diverse pool of low frequency ALVE integrations. Further work is needed to characterise the evolutionary and immunological roles of ALVE within these populations, but our observations are typical of a role in ERV-derived immunity. Six novel ALVE were identified within genes which warrant further investigation to determine their specific effects on the host. Identification of ALVE with detrimental effects on productivity may help guide local breeding programmes. 
In addition, although ALVE are typically negatively associated with productivity in a commercial setting, their potential role in defence against exogenous ALV may provide an overall net benefit in the productivity of indigenous chickens.

\section{Supplementary information}

Supplementary information accompanies this paper at https://doi. org/10.1186/s12711-020-00548-4.

Additional file 1. All ALVE matrix. This document contains a list of all identified ALVE ordered according to their Galgal5 coordinates, with their name, target site duplication, and previous ambiguous names, where applicable. The genotype of each ALVE is indicated by 0 for homozygous wild type, 0.5 for heterozygotes and 1 for ALVE insertion homozygotes. This file also includes all Ethiopian and Nigerian regional abbreviations.

Additional file 2: Table S1. Sampled populations and their identified ALVE diversity. The table includes how many individuals were sampled in each site, the total number of different ALVE identified in those birds, and the number of those which were only found in that region. Table S2. Individual chicken samples selected for PCR validation of bioinformatically detected sites by obsERVer. This table includes the 20 randomly selected ALVE to validate the findings of obsERVer, the selected individuals and their bioinformatically-predicted genotype. Table S3. Diagnostic ALVE PCR assays designed for obsERVer validation. This table lists the PCR primers for the obsERVer validation and the predicted and product length for each allele. Table S4. ALVE distribution relative to coding features and randomly simulated integrations. This table lists the observed genomic distribution of ALVE relative to coding features when compared with a model of random integration. These values support Fig. 3. Table S5. ALVE distribution relative to coding feature regions and randomly simulated integrations. This table pairs with Table S4 and shows the observed and simulated values for ALVE integration within exons, UTRs and introns.

Additional file 3: Figure S1. Agilent 4200 TapeStation results for 20 diagnostic assays used for the validation of the bioinformatically detected ALVE integrations by obsERVer. PCR results for 20 ALVE detected by obsERVer selected to validate the bioinformatically detected integrations.

Additional file 4: Figure S2. Phylogeny of sampled birds on ALVE genotype. Dendrogram of all individuals based on their ALVE content. Figure 2 indicates population structure in a similar manner, but this supplementary figure labels each individual dataset.

\section{Acknowledgements}

The authors would like to thank Edinburgh Genomics (Edinburgh, UK) for generating the whole-genome sequence data, and Almas Gheyas for her technical assistance in the early bioinformatic processing of the sequencing data.

\section{Authors' contributions}

JS conceived the initial concept for the study. AK, OB, ASA, TD and OH collected the samples used in this study. ASM performed the bioinformatic analyses and prepared the manuscript. KM performed the PCR validation. All authors read and approved the final manuscript.

\section{Funding}

This work was funded by the Biotechnology and Biological Sciences Research Council (BBSRC) as part of an Impact Accelerator Award (BB/GCRF-IAA/25), contributing to the larger Bill and Melinda Gates (BMGF) funded project (OPP1127286) awarded to the Centre for Tropical Livestock Genetics and Health.

\section{Availability of data and materials}

Additional file 1 accompanying this manuscript contains a complete list of the ALVE with their locations and the individuals in which they were identified. The obsERVer pipeline is freely available on GitHub (https://github.com/andre wstephenmason/obsERVer). The Galgal5 reference genome is available on
GenBank (GCF_000002315.4). WGS data is available from the authors upon reasonable request. All transfer of samples, data analysis and sharing complies with the principles set out in the Nagoya Protocol.

Ethics approval and consent to participate

No ethical consent was required for this work.

\section{Consent for publication}

Not applicable.

\section{Competing interests}

The authors declare they have no competing interests.

\section{Author details}

${ }^{1}$ The University of York, York YO10 5DD, UK. ${ }^{2}$ The Roslin Institute and Royal (Dick) School of Veterinary Studies, The University of Edinburgh, Easter Bush, Midlothian EH25 9RG, UK. ${ }^{3}$ LiveGene-CTLGH, International Livestock Research Institute (ILRI), Addis Ababa, Ethiopia. ${ }^{4}$ Addis Ababa University, Addis Ababa, Ethiopia. ${ }^{5}$ African Chicken Genetic Gains, Department of Animal Sciences, Obafemi Awolowo, Ile Ife, Osun, Nigeria. ${ }^{6}$ School of Life Sciences, The University of Nottingham, University Park, Nottingham NG7 2RD, UK. ${ }^{7}$ University of Anbar, Ramadi, Anbar, Iraq.

Received: 19 September 2019 Accepted: 26 May 2020

Published online: 01 June 2020

\section{References}

1. Doolittle RF, Feng DF, Johnson MS, McClure MA. Origins and evolutionary relationships of retroviruses. Q Rev Biol. 1989;64:1-30.

2. Patel MR, Emerman M, Malik HS. Paleovirology_ghosts and gifts of the past. Curr Opin Virol. 2011;1:304-9.

3. Stoye JP. Studies of endogenous retroviruses reveal a continuing evolutionary saga. Nat Rev Microbiol. 2012:10:395-406.

4. Katz RA, Skalka AM. Generation of diversity in retroviruses. Annu Rev Genet. 1990;24:409-45.

5. Magiorkinis G, Gifford RJ, Katzourakis A, De Ranter J, Belshaw R. Env-less endogenous retroviruses are genomic superspreaders. Proc Natl Acad Sci USA. 2012;109:7385-90.

6. Stoye JP. Endogenous retroviruses: still active after all these years? Curr Biol. 2001:11:R914-6.

7. Venugopal K. Avian leukosis virus subgroup J: a rapidly evolving group of oncogenic retroviruses. Res Vet Sci. 1999;67:113-9.

8. Liu C, Zheng S, Wang Y, Jing L, Gao H, Gao Y, et al. Detection and molecular characterization of recombinant avian leukosis viruses in commercial egg-type chickens in China. Avian Pathol. 2011;40:269-75.

9. Henzy JE, Gifford RJ, Johnson WE, Coffin JM. A novel recombinant retrovirus in the genomes of modern birds combines features of avian and mammalian retroviruses. J Virol. 2014:88:2398-405.

10. Varela M, Spencer TE, Palmarini M, Arnaud F. Friendly viruses. Ann NY Acad Sci. 2009;1178:157-72.

11. Ito J, Watanabe S, Hiratsuka T, Kuse K, Odahara Y, Ochi H, et al. Refrex-1, a soluble restriction factor against feline endogenous and exogenous retroviruses. J Virol. 2013;87:12029-40.

12. Kozak CA. Origins of the endogenous and infectious laboratory mouse gammaretroviruses. Viruses. 2014;7:1-26.

13. Lepperdinger G, Müllegger J, Kreil G. Hyal2_less active, but more versatile? Matrix Biol. 2001;20:509-14.

14. Jadin L, Wu X, Ding H, Frost Gl, Onclinx C, Triggs-Raine B, et al. Skeletal and hematological anomalies in HYAL2-deficient mice: a second type of mucopolysaccharidosis IX? FASEB J. 2008;22:4316-26.

15. Aswad A, Katzourakis A. Paleovirology and virally derived immunity. Trends Ecol Evol. 2012;27:627-36.

16. Katzourakis A, Gifford RJ. Endogenous viral elements in animal genomes. PLoS Genet. 2010;6:e1001191.

17. Hurst T, Magiorkinis G. Activation of the innate immune response by endogenous retroviruses. J Gen Virol. 2015:96:1207-18.

18. Mason AS, Fulton JE, Hocking PM, Burt DW. A new look at the LTR retrotransposon content of the chicken genome. BMC Genomics. 2016;17:688. 
19. Warren WC, Hillier LW, Tomlinson C, Minx P, Kremitzki M, Graves T, et al. A new chicken genome assembly provides insight into avian genome structure. G3 (Bethesda). 2017;7:109-17.

20. Borysenko L, Stepanets V, Rynditch AVV. Molecular characterization of full-length MLV-related endogenous retrovirus ChiRV1 from the chicken, Gallus gallus. Virology. 2008;376:199-204.

21. Payne LN, Nair $V$. The long view: 40 years of avian leukosis research. Avian Pathol. 2012:41:11-9.

22. Frisby DP, Weiss RA, Roussel M, Stehelin D. The distribution of endogenous chicken retrovirus sequences in the DNA of galliform birds does not coincide with avian phylogenetic relationships. Cell. 1979;17:623-34.

23. Robinson HL, Astrin SM, Senior AM, Salazar FH. Host susceptibility to endogenous viruses: defective, glycoprotein-expressing proviruses interfere with infections. J Virol. 1981;40:745-51.

24. Smith EJ, Fadly AM, Levin I, Crittenden LB. The influence of ev6 on the immune response to avian leukosis virus infection in rapid-feathering progeny of slow- and rapid-feathering dams. Poult Sci. 1991;70:1673-8.

25. Crittenden LB, Smith EJ, Fadly AM. Influence of endogenous viral (ev) gene expression and strain of exogenous avian leukosis virus (ALV) on mortality and ALV infection and shedding in chickens. Avian Dis. 1984;28:1037-56.

26. Fox W, Smyth JR Jr. The effects of recessive white and dominant white genotypes on early growth rate. Poult Sci. 1985;64:429-33.

27. Kuhnlein U, Sabour M, Gavora JS, Fairfull RW, Bernon DE. Influence of selection for egg production and Marek's disease resistance on the incidence of endogenous viral genes in White Leghorns. Poult Sci. 1989;68:1161-7.

28. Gavora JS, Kuhnlein U, Crittenden LB, Spencer JL, Sabour MP. Endogenous viral genes: association with reduced egg production rate and egg size in White Leghorns. Poult Sci. 1991;70:618-23.

29. Rutherford K, Meehan CJ, Langille MGI, Tyack SG, McKay JC, McLean NL, et al. Discovery of an expended set of avian leukosis subroup E proviruses in chickens using Vermillion, a novel sequence capture and analysis pipeline. Poult Sci. 2016;95:2250-8.

30. Mason AS. The abundance and diversity of endogenous retroviruses in the chicken genome. Ph.D. thesis, University of Edinburgh. 2018.

31. Martin M. Cutadapt removes adapter sequences from high-throughput sequencing reads. EMBnet J. 2011;17:10-2.

32. Andrews S. FastQC. "A quality control tool for high throughput sequence data." 2010. https://www.bioinformatics.babraham.ac.uk/projects/fastqc/. Accessed 23 Oct 2017.

33. Krueger F. Trim Galore. "A wrapper tool around Cutadapt and FastQC to consistently apply quality and adapter trimming to FastQ files, with some extra functionality for Mspl-digested RRBS-type (Reduced Representation Buisulfite-Seq) libraries. 2013. https://www.bioinformatics.babraham. ac.uk/projects/trim_galore/. Accessed 23 Oct 2017.

34. Thorvaldsdóttir H, Robinson JT, Mesirov JP. Integrative genomics viewer (IGV): high-performance genomics data visualization and exploration. Brief Bioinform. 2013;14:178-92.

35. Untergasser A, Cutcutache I, Koressaar T, Ye J, Faircloth BC, Remm M, et al. Primer3 - new capabilities and interfaces. Nucleic Acids Res. 2012;40:e115.

36. Elferink MG, van As P, Veenendaal T, Crooijmans RP, Groenen MA. Regional differences in recombination hotpsots between two chicken populations. BMC Genet. 2010;11:11.
37. Li H. Aligning sequence reads, clone sequences and assembly contigs with BWA-MEM. 2013; arXiv.1303.3997v2 [q-bio.GN].

38. van der Maaten LJP. Using t-SNE. J Mach Learn Res. 2008;9:2579-605.

39. Benkel BF. Locus-specific diagnostic tests for endogenous avian leukosistype viral loci in chickens. Poult Sci. 1998;77:1027-35.

40. Bacon LD, Smith E, Crittenden LB, Havenstein GB. Association of the slow feathering (K) and an endogenous viral (ev21) gene on the Z chromosome of chickens. Poult Sci. 1988;67:191-7.

41. Elferink MG, Vallée AAA, Jungerius AP, Crooijmans RP, Groenen MA. Partial duplication of the PRLR and SPEF2 genes at the late feathering locus in chicken. BMC Genomics. 2008;9:391.

42. Bu G, Huang G, Fu H, Li J, Huang S, Wang Y. Characterization of the novel duplicated PRLR gene at the late-feathering K locus in Lohmann chickens. J Mol Endocrinol. 2013;51:261-76.

43. Smith EJ, Fadly AM, Crittenden LB. Interactions between endogenous virus loci ev6 and ev21:: 1 Immune response to exogenous avian leukosis virus infection. Poult Sci. 1990;69:1244-50.

44. Gavora JS, Spencer JL, Benkel B, Gagnon C, Emsley A, Kulenkamp A. Endogenous viral genes influence infection with avian leukosis virus. Avian Pathol. 1995;24:653-64.

45. Levin E, Smith EJ. Molecular analysis of endogenous virus ev21-slow feathering complex of chickens. 1. Cloning of proviral-cell junction fragment and unoccupied integration site. Poult Sci. 1990;69:2017-26.

46. Takenouchi A, Toshishige M, Ito N, Tsudzuki M. Endogenous viral gene ev21 is not responsible for the expression of late feathering in chickens. Poult Sci. 2018;97:403-11.

47. Narezkina A, Taganov KD, Litwin S, Stoyanova R, Hayashi J, Seeger C, et al. Genome-wide analyses of avian sarcoma virus integration sites. J Virol. 2004;78:11656-63.

48. Serrao E, Ballandras-Colas A, Cherepanov P, Maertens GN, Engelman AN Key determinants of target DNA recognition by retroviral intasomes. Retrovirology. 2015;12:39.

49. Grawenhoff J, Engelman AN. Retroviral integrase protein and intasome nucleoprotein complex structures. World J Biol Chem. 2017;26:32-44.

50. Park M, Kim S, Kim Y, Chung YH. ALS2CR7 (CDK15) attenuates TRAIL induced apoptosis by inducing phosphorylation of survivin Thr34. Biochem Biophys Res Commun. 2014;450:129-34.

51. Lai CK, Gupta N, Wen X, Chih B, Peterson AS, Bazan JF, et al. Functional characterizaton of putative cilia genes by high-content analysis. Mol Biol Cell. 2011;22:1104-19.

52. Rifas L, Weitzmann M. A novel T cell cytokine, secreted osteoclastogenic factor of activated T cells, induces osteoclast formation in a RANKL-independent manner. Arthritis Rheumat. 2009;60:3324-35.

53. Kelly D, Kotliar M, Woo V, Jagannathan S, Whitt J, Moncivaiz J. Microbiotasensitive epigenetic signature predicts inflammation in Crohn's disease. JCl Insight. 2018;3:122104.

54. Ostuni A, Lara P, Armentano M, Miglionico R, Salvia AM, Mönnich M, et al. The hepatitis Bx antigen anti-apoptotic effector URG7 is localized to the endoplasmic reticulum. FEBS Lett. 2013;587:3058-62.

\section{Publisher's Note}

Springer Nature remains neutral with regard to jurisdictional claims in published maps and institutional affiliations.

Ready to submit your research? Choose BMC and benefit from

- fast, convenient online submission

- thorough peer review by experienced researchers in your field

- rapid publication on acceptance

- support for research data, including large and complex data types

- gold Open Access which fosters wider collaboration and increased citations

- maximum visibility for your research: over $100 \mathrm{M}$ website views per year

At BMC, research is always in progress.

Learn more biomedcentral.com/submissions 